\title{
Sinonasal Inverted Papilloma: Evaluating the Effectiveness of Prediction of the Site of Attachment based on Computed Tomography Scans
}

\author{
Anna Salwa
}

\begin{abstract}
Sinonasal inverted papilloma is a benign neoplasm of epithelial origin, which represents 0.5 to $4 \%$ of all sinonasal tumors. Despite the histological benign nature of this type of tumor, it has a high rate of recurrence after surgical resection and significant malignant potential. Sinonasal inverted papilloma is treated as a locally aggressive tumor. The aggressive surgical approaches, such as en bloc resection via external excision or extensive mucosal stripping of the ipsilateral sinuses are replaced by less invasive intranasal endoscopic approaches. This creates a need for a very accurate preoperative assessment of the location of the tumor on computed tomography (CT) scan and identifies the location of the primary changes. Determination of the primary location of inverted papilloma is especially important for a radical resection of the tumor, because the tumor recurs mostly in the same location as the primary lesion. The aim of this study was to determine the relationship between changes in the preoperative CT scan of the paranasal sinuses and the location of papilloma by histological examination of surgical material.

In this study, we evaluated the changes in the preoperative CT scans in the form of focal hyperostosis and erosion of the walls of the paranasal sinuses. Examined groups consisted of 12 people diagnosed with inverted papilloma and 15 peoples diagnosed with preinverted papilloma.

Focal hyperostosis occurred in $83 \%$ of cases of inverted papilloma. Their location corresponded to $90 \%$ of the primal attachment of the tumor. Among the preinverted papillomas, focal hyperostosis occurred in less than $7 \%$ of the cases and did not correspond to the location of papilloma inverted. The analysis of remodeling and erosion of bone walls of the sinuses showed their presence in $67 \%$ of cases of inverted papilloma and $40 \%$ of cases of preinverted papilloma. However, their overlap with the location of the tumor was $50 \%$ for the inverted papilloma and $33 \%$ for the preinverted papilloma.

Erosion and sinus bone destruction do not coincide closely with the primary location of the papilloma, because it is more the result of bone compression by the growing mass than bone invasion. The sites of focal hyperostosis closely coincide with the place of origin of the inverted papilloma, but the pathophysiological mechanism of this phenomenon is not fully understood. The relationship between the sites of focal hyperostosis and the location of the primary tumor can be used when planning surgery for accurate resection.
\end{abstract}

Keywords: Computed tomography, Inverted papilloma, Site of attachment.

MD

Department of Otolaryngology, 5th Military Hospital, Krakow Poland

Corresponding Author: Anna Salwa, MD, Department of Otolaryngology, 5th Military Hospital, Krakow, Poland, e-mail: anna.salwa.05@gmail.com
How to cite this article: Salwa A. Sinonasal Inverted PapilIoma: Evaluating the Effectiveness of Prediction of the Site of Attachment based on Computed Tomography Scans. Clin Rhinol An Int J 2016;9(2):84-86.

\section{Source of support: Nil}

Conflict of interest: None

\section{INTRODUCTION}

Inverted papilloma is a common benign epithelial tumor and represents 0.5 to $4 \%$ of all sinonasal tumors. Inverted papilloma of the nasal cavity and paranasal sinuses arises from embryonic remnants of the Schneiderian mucosa, which separates the mucosa of the nose and paranasal sinuses. The proliferation of the squamous epithelium and its ingrown proliferation into the stroma can be seen in the histology. ${ }^{1-3}$ Inverted papilloma shows locally aggressive growth with a tendency to tissue destruction and recurrences. Papilloma is associated with squamous cell carcinoma in approximately $10 \%$ of the cases. ${ }^{1,4,5}$ The treatment of inverted papilloma is exclusively surgical. The choice of surgical technique depends on the location and size of the changes. This creates a need for very accurate preoperative assessment of the location of the tumor on computed tomography (CT) scans. ${ }^{1,6}$ Determination of the primary location of the inverted papilloma is especially important for a radical resection of the tumor, because the tumor recurs in the same location as the primary lesion. ${ }^{1,6,7}$ The aim of this study was to determine the relationship between the changes in preoperative CT scans of the paranasal sinuses with the placement of the papilloma by histological examination of intraoperatively collected samples.

\section{MATERIALS AND METHODS}

Groups of subjects consisted of 12 people diagnosed with inverted papilloma and 15 people diagnosed with preinverted papilloma (hyperplastic proliferation of the squamous epithelium without ingrowth into the stroma). All patients had preoperative CT scans of the paranasal sinus. The CT scan protocol for the study employed a thin-cut $(1 \mathrm{~mm})$ axial and/or coronal scan without administering a contrast agent. The severity of bone erosion and focal 


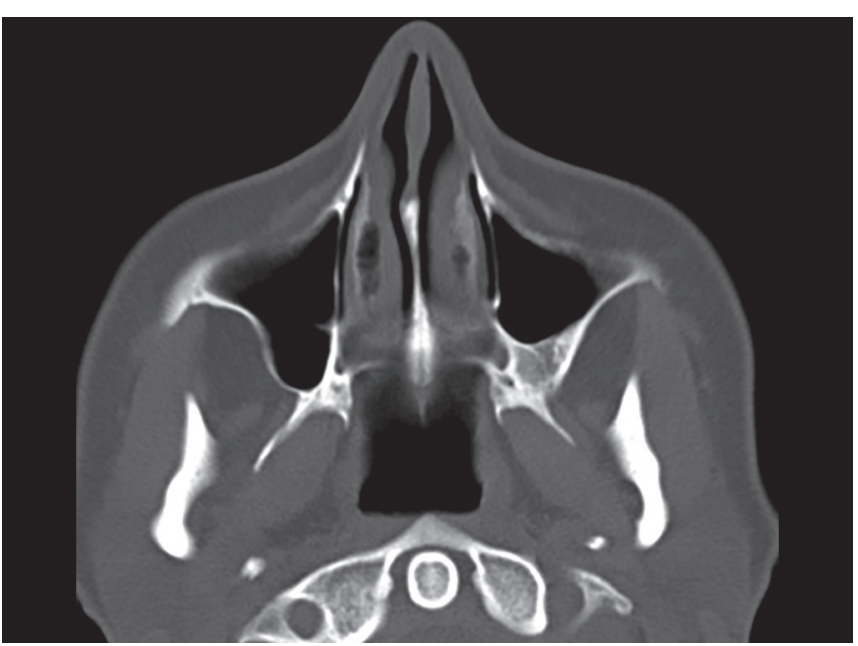

Fig. 1: Focal hyperostosis

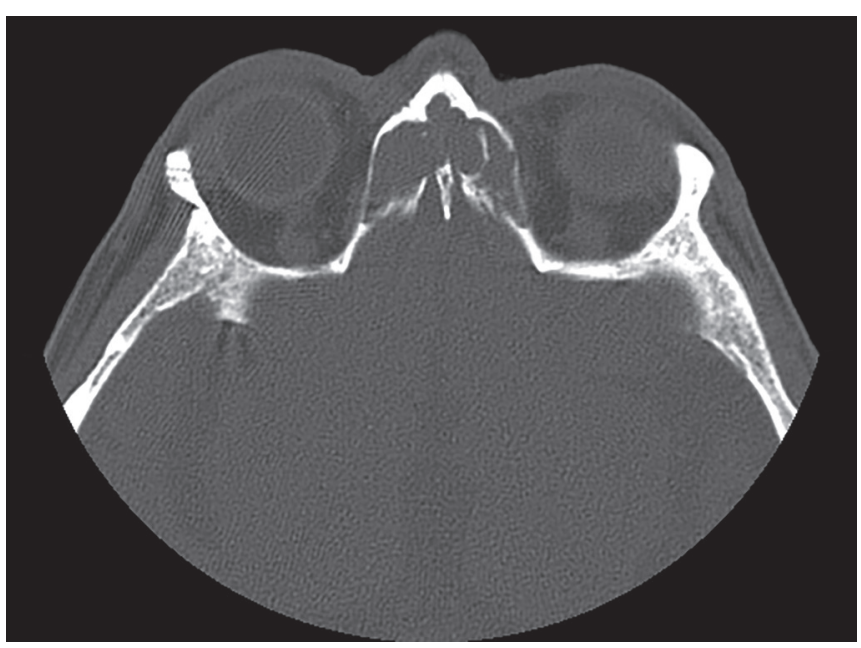

Fig. 3: Focal hyperostosis

hyperostosis (neo-osteogenesis) was measured using a three-point scale (1 - small, visible on detailed evaluation; 2 - moderate, highly visible; 3 - very intense). The average age in the group of inverted papilloma was 57.5 years and in the group of preinverted papilloma, 51.6 years. Both groups were significantly dominated by men (Figs 1 to 3 ).

\section{RESULTS}

Changes in paranasal sinuses in the cases of inverted papilloma were in most cases located on one side; only in two cases, they were bilateral (16\%). In the cases of preinverted papilloma, bilateral changes occurred in $26 \%$ of cases. Focal hyperostosis occurred in $83 \%$ of cases of inverted papilloma. Their location corresponded to $90 \%$ of the primal attachment of the tumor. Among the preinverted papilloma group, the focal hyperostosis occurred in $6.6 \%$ of cases and did not correspond to the location of papilloma inverted. The intensity of the process of neo-osteogenesis (focal hyperostosis) in the inverted papilloma group was evaluated mainly as moderate $(60 \%$

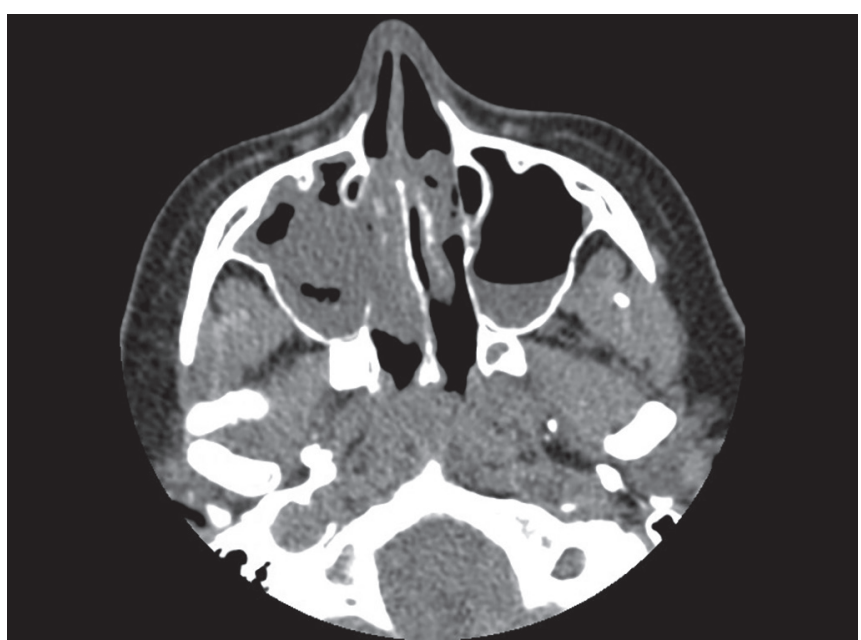

Fig. 2: The erosion of bone

of cases) (small - 30\%, very intense $-10 \%$ ). If the focal hyperostosis occurred in a group of preinverted papilloma, it was rated as small.

The analysis of remodeling and erosion of bone walls of the sinuses showed their presence in $66.6 \%$ of cases of inverted papilloma and $40 \%$ of cases of preinverted papilloma. However, their overlap with the location of the tumor was 50\% for inverted papilloma and 33\% for preinverted papilloma (Graphs 1 and 2). The degree of erosion of the bones was assessed in a group of inverted papilloma as follows: Small $-62.5 \%$, moderate $-12.5 \%$, and intense $-25 \%$. Among the preinverted severity of rated on: Small $-66.6 \%$, moderate $-33.3 \%$.

\section{DISCUSSION}

Aggressive surgical approaches, such as en bloc resection via external excision or extensive mucosal stripping of the ipsilateral sinuses are replaced by less invasive intranasal endoscopic approaches. This creates a need for very accurate preoperative assessment of the location of

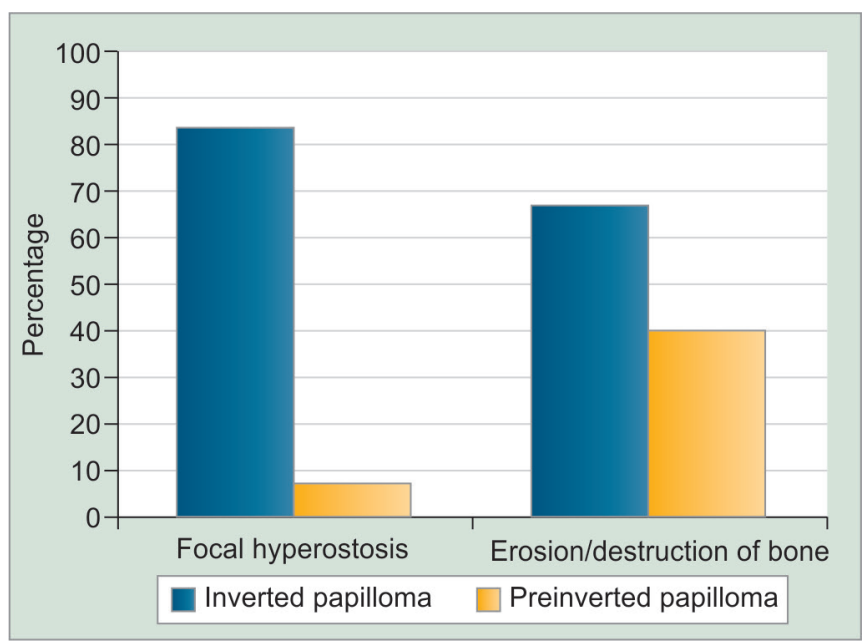

Graph 1: Bone changes in preoperative CT scans 


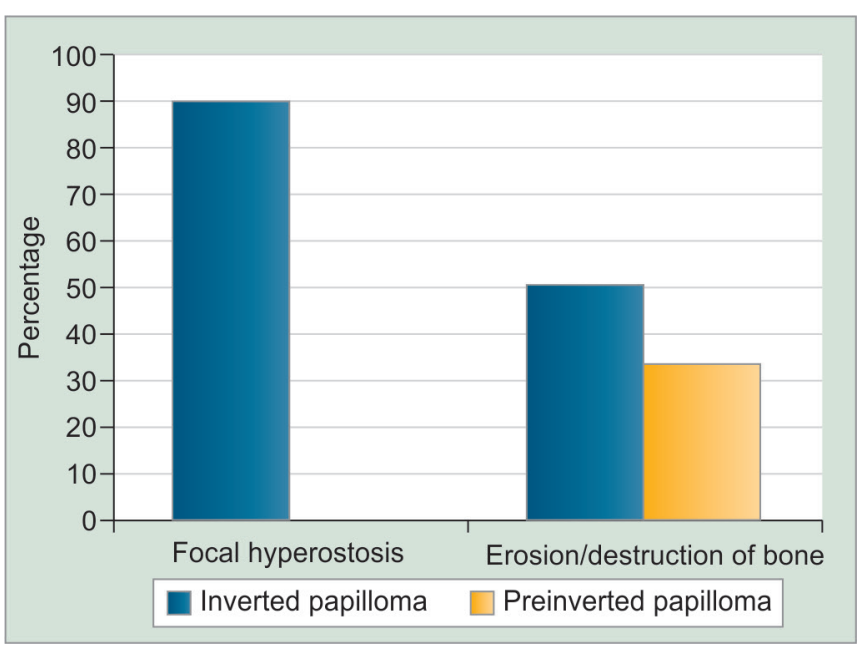

Graph 2: The accuracy of predicting the location of papilloma

the tumor on CT scan and identifies the location of the primary changes. ${ }^{1,6,8,9}$ Determining the primary location of inverted papilloma is especially important for a radical resection of the tumor, because the tumor recurs mostly in the same location as the primary lesion. ${ }^{1,6,8-10}$ The sites of focal hyperostosis closely coincide with the place of origin of the inverted papilloma, but the pathophysiological mechanism of this phenomenon is not fully understood. ${ }^{1,6,11}$ The opinions of the authors are divided and tend toward a mechanism involving the activation of osteoblast by cytokines produced during an inflammatory response or increased bone formation at the site of attachment of the tumor to the bone as a result of increased vascularity of this site. ${ }^{1,6,7}$ Therefore, only the local neo-osteogenesis is important in determining the attachment site. Extensive bone formation in the form of thickening of the walls is a feature of chronic sinusitis, which could interfere with the results. ${ }^{1,2}$ Erosion and sinus bone destruction do not coincide closely with the primary location of the papilloma, because it is more the result of bone compression by the growing mass than bone invasion. ${ }^{1,8,10}$ The large degree of erosion and destruction of bony walls is more characteristic of the presence of squamous cell carcinoma. The preinverted type of hyperplasia is not associated with neo-osteogenesis, and the bone erosion of the walls is less severe. ${ }^{8,9}$ In the author's opinion, it is caused by lack of invasion in the stroma, which provokes an inflammatory response in the stroma and affects the aforementioned processes.

\section{CONCLUSION}

The relationship between the sites of focal hyperostosis and the location of the primary tumor can be used when planning surgery for accurate resection and reduce the likelihood of local recurrence.

Erosion and destruction of bony walls is not characteristic of the presence of sinonasal inverted papilloma.

\section{REFERENCES}

1. Bhalla RK, Wright ED. Predicting the site of attachment of sinonasal inverted papilloma. Rhinology 2009 Dec;47(4): 345-348.

2. ŁukomskiM,Obrębka R,Starska K, Pietruszewska W, DurkoM, Pajor A, Gryczyński M, Józefowicz-Korczyńska M. Results of treatment papilloma of nasal cavity and paranasal sinuses. Otolaryngol Pol 2008;62(5):574-577.

3. Kim DY, Hong SL, Lee CH, Jin HR, Kang JM, Lee BJ, Moon IJ, Chung SK, Rha KS, Cho SH, et al. Inverted papilloma of the nasal cavity and paranasal sinuses: a Korean multicenter study. Laryngoscope 2012 Mar;122(3):487-494.

4. Wassef SN, Batra PS, Barnett S. Skull base inverted papilloma: a comprehensive review. ISRN Surg 2012;2012:175903.

5. Saha SN, Ghosh A, Sen S, Chandra S, Biswas D. Inverted papilloma: a clinicopathological dilemma with special reference to recurrence and malignant transformation. Indian J Otolaryngol Head Neck Surg 2010 Oct;62(4):354-359.

6. Lee DK, Chung SK, Dhong HJ, Kim HY, Kim HJ, Bok KH. Focal hyperostosis on CT of sinonasal inverted papilloma as a predictor of tumor origin. AJNR Am J Neuroradiol 2007 Apr;28(4):618-621.

7. Chiu AG, Jackman AH, Antunes MB, Feldman MD, Palmer JN. Radiographic and histologic analysis of the bone underlying inverted papillomas. Laryngoscope 2006 Sep;116(9): 1617-1620.

8. Woodruff WW, Vrabec DP. Inverted papilloma of the nasal vault and paranasal sinuses: spectrum of CT findings. AJR Am J Roentgenol 1994 Feb;162(2):419-423.

9. Dammann F, Pereira P, Laniado M, Plinkert P, Löwenheim H, Claussen CD. Inverted papilloma of the nasal cavity and the paranasal sinuses: using CT for primary diagnosis and follow-up. AJR Am J Roentgenol 1999 Feb;172(2):543-548.

10. Sham CL, King AD, van Hasselt A, Tong MC. The roles and limitations of computed tomography in the preoperative assessment of sinonasal inverted papillomas. Am J Rhinol 2008 Mar-Apr;22(2):144-150.

11. Nakamaru Y, Fujima N, Takagi D, Tsukahara A, Yoshida D, Fukuda S. Prediction of the attachment site of sinonasal inverted papillomas by preoperative imaging. Ann Otol Rhinol Laryngol 2014 Jul;123(7):468-474. 